Comment. Math. Helv. 79 (2004) 826-839 0010-2571/04/040826-14

DOI $10.1007 / \mathrm{s} 00014-004-0812-2$
(C) 2004 Birkhäuser Verlag, Basel

Commentarii Mathematici Helvetici

\title{
Rigid resolutions and big Betti numbers
}

\author{
Aldo Conca, Jürgen Herzog and Takayuki Hibi
}

\begin{abstract}
The Betti-numbers of a graded ideal $I$ in a polynomial ring and the Betti-numbers of its generic initial ideal $\operatorname{Gin}(I)$ are compared. In characteristic zero it is shown that if these Betti-numbers coincide in some homological degree, then they coincide in all higher homological degrees. We also compare the Betti-numbers of componentwise linear ideals which are contained in each other and have the same Hilbert polynomial.
\end{abstract}

Mathematics Subject Classification (2000). 13D02, 13P10, 13D40, 13 A02.

Keywords. Free resolutions, generic initial ideals, componentwise linear ideals.

\section{Introduction}

Let $K$ be a field of characteristic 0 and $S=K\left[x_{1}, \ldots, x_{n}\right]$ the polynomial ring over $K$ with graded maximal ideal $\mathfrak{m}=\left(x_{1}, \ldots, x_{n}\right)$. Denote by $\beta_{i}(M)=\operatorname{Tor}_{i}^{S}(K, M)$ the $i$ th Betti number of a finitely generated graded module $M$ and by $\operatorname{Gin}(I)$ the generic initial ideal of a graded ideal $I$ with respect to the reverse lexicographical order.

In this paper we answer (positively) a question raised by the first author in [8]. We prove that if a graded ideal $I \subset S$ has $\beta_{i}(I)=\beta_{i}(\operatorname{Gin}(I))$ for some $i$, then $\beta_{k}(I)=\beta_{k}(\operatorname{Gin}(I))$ for all $k \geq i$, see Corollary 2.4. For $i=0$, this theorem was first proved by Aramova, Herzog and Hibi [2]. More generally, we show that the same statement holds if $\operatorname{Gin}(I)$ is replaced by either any generic initial ideal of $I$ or by the lex-segment ideal associated with $I$.

Given a finitely generated graded $S$-module $M$, a generic sequence of linear forms $y_{1}, \ldots, y_{n}$ and an integer $p, 1 \leq p \leq n$, we define the generic annihilator number $\alpha_{p}(M)$ of $M$ to be $\operatorname{dim}_{K}\left(\left(y_{1}, \ldots, y_{p-1}\right) M:_{M} y_{p} /\left(y_{1}, \ldots, y_{p-1}\right) M\right.$ and the generic Koszul homology $H_{i}\left(y_{1}, \ldots, y_{p} ; M\right)$ to be the $i$ th-homology of the Koszul complex over $M$ with respect to $y_{1}, \ldots, y_{p}$.

In the first section of this paper we show that there is an upper bound for the Betti numbers of $M$ in terms of the generic annihilator numbers of $M$. We show in Theorem 1.5, that among other equivalent conditions, this upper bound is achieved for all $i$ if and only if $\mathfrak{m} H_{i}\left(y_{1}, \ldots, y_{p} ; M\right)=0$ for all $i>0$ and $p=1, \ldots, n$. 
The above mentioned Corollary 2.4 is a consequence of the more general Theorem 2.3, proved in Section 2, which says that if the $i$ th Betti number of $M$ achieves the upper bound given by the generic annihilator numbers, then the upper bound is also achieved for $j$ th Betti numbers with $j>i$. For the proof of this theorem the following interesting annihilation property of Koszul homology is required: suppose that for a generic sequence $y=y_{1}, \ldots, y_{n}$ of linear forms and some $i$ one has $\mathfrak{m} H_{i}\left(y_{1}, \ldots, y_{p} ; M\right)=0$ for all $p$ then $\mathfrak{m} H_{k}\left(y_{1}, \ldots, y_{p} ; M\right)=0$ for all $k \geq i$ and for all $p$.

In the last section of the paper we show that if two componentwise linear ideals $I \subset J \subset S$ have the same Hilbert polynomial then $\beta_{i}(J) \leq \beta_{i}(I)$ for all $i$, see Theorem 3.2. This theorem was inspired by a question of Eisenbud and Huneke: suppose $\operatorname{char}(K)=0$ and $I$ is a graded $\mathfrak{m}$-primary ideal in $S$ with $I \subset \mathfrak{m}^{d}$ for some $d$. Is it then true that the number of generators of $\operatorname{Gin}(I)$ is greater than or equal to the number of generators of $\mathfrak{m}^{d}$ ? As an application of Theorem 3.2 we show in Corollary 3.4 that this is indeed the case. Moreover, we show that $\operatorname{Gin}(I)$ and $\mathfrak{m}^{d}$ have the same number of generators if and only if $I+(y)=\mathfrak{m}^{d}+(y)$ for a generic linear form $y$, see 3.4 .

Not all results in this paper require that $K$ is a field of characteristic 0. However in those results which refer to generic initial ideals we need this hypothesis, otherwise they are false.

We would like to thank MSRI in Berkeley for its hospitality while part of the research for this paper was carried out. The results and the examples presented in this paper have been inspired and suggested by computations performed by the computer algebra system $\mathrm{CoCoA}$ [7]. We would also like to thank Giulio Caviglia for useful discussions regarding 3.6.

\section{An upper bound for Betti numbers}

Let $K$ be an arbitrary field, unless otherwise stated, $S=K\left[x_{1}, \ldots, x_{n}\right]$ the polynomial ring in $n$ variables over $K$ with each $\operatorname{deg} x_{i}=1, \mathfrak{m}=\left(x_{1}, \ldots, x_{n}\right)$ the graded maximal ideal and $M$ a finitely generated graded $S$-module.

The $S$-modules $\operatorname{Tor}_{i}^{S}(K, M)$ are finitely generated graded $K$-vector spaces. The numbers

$$
\beta_{i}(M)=\operatorname{dim}_{K} \operatorname{Tor}_{i}^{S}(K, M)
$$

are called the Betti numbers of $M$. They are invariant under base field extensions, so that, without any restrictions, we may assume that the base field is infinite. We will consider also the graded Betti number $\beta_{i j}$ defined as the dimension of the degree $j$ component of $\operatorname{Tor}_{i}^{S}(K, M)$.

We want to relate the Betti numbers of $M$ to another sequence of numbers, $\alpha_{1}(M), \alpha_{2}(M), \cdots$, which we call the generic annihilator numbers of $M$.

The property of a linear form to have a minimal finite length annihilator with respect to a module is a Zariski-open non-empty condition on the space of linear 
forms. We call a linear form with this property generic (with respect to the module). To avoid endless repetitions we tacitly assume that modules to which generic refers are clear from the context.

Let $y=y_{1}, \ldots, y_{n}$ be generic linear forms. Then

$$
A_{p}=\left(y_{1}, \ldots, y_{p-1}\right) M:_{M} y_{p} /\left(y_{1}, \ldots, y_{p-1}\right) M
$$

is a module of finite length. We set

$$
\alpha_{p}(M)=\operatorname{dim}_{K} A_{p} .
$$

Generic annihilators and generic annihilator numbers were first considered in a paper by Trung [18] in the context of local cohomology, and were subsequently used in other contexts, for example in [1] and [19]. We denote by $H_{i}(p, M)$ the Koszul homology $H_{i}\left(y_{1}, \ldots, y_{p} ; M\right)$ of the partial sequence $y_{1}, \ldots, y_{p}$, and set $h_{i}(p, M)=$ $\operatorname{dim}_{K} H_{i}(p, M)$. If there is no danger of confusion, we simply write $\beta_{i}, \alpha_{i}, H_{i}(p)$ and $h_{i}(p)$ for $\beta_{i}(M), \alpha_{i}(M), H_{i}(p, M)$ and $h_{i}(p, M)$ respectively.

Attached with $y$ there are long exact sequences

$$
\begin{gathered}
\cdots \longrightarrow H_{i}(p-1) \stackrel{\varphi_{i, p-1}}{\longrightarrow} H_{i}(p-1) \longrightarrow H_{i}(p) \longrightarrow H_{i-1}(p-1) \\
\cdots \longrightarrow H_{0}(p-1) \stackrel{\varphi_{0, p-1}}{\longrightarrow} H_{0}(p-1) \longrightarrow 0 .
\end{gathered}
$$

Here $\varphi_{i, p-1}: H_{i}(p-1) \rightarrow H_{i}(p-1)$ is the map given by multiplication with $\pm y_{p}$. Note that $A_{p}$ is the Kernel of the map $\varphi_{0, p-1}$. We conclude that

$$
h_{1}(p)=h_{1}(p-1)+\alpha_{p}-\operatorname{dim}_{K} \operatorname{Im} \varphi_{1, p-1}
$$

for all $p$ and

$$
h_{i}(p)=h_{i}(p-1)+h_{i-1}(p-1)-\operatorname{dim}_{K} \operatorname{Im} \varphi_{i, p-1}-\operatorname{dim}_{K} \operatorname{Im} \varphi_{i-1, p-1}
$$

for all $p$ and $i>1$. With the notation introduced we have:

Proposition 1.1. Given integers $1 \leq i \leq p$ we define the set

$$
A_{i, p}=\left\{(a, b) \in \mathbb{N}^{2}: 1 \leq b \leq p-1 \text { and } \max (i-p+b, 1) \leq a \leq i\right\} .
$$

We have

(a) $h_{i}(p) \leq \sum_{j=1}^{p-i+1}\left(\begin{array}{c}p-j \\ i-1\end{array}\right) \alpha_{j}$ for all $i \geq 1$ and $p \geq 1$.

(b) For given $i \geq 1$ and $p \geq 1$ the following conditions are equivalent:

(i) $h_{i}(p)=\sum_{j=1}^{p-i+1}\left(\begin{array}{c}p-j \\ i-1\end{array}\right) \alpha_{j}$

(ii) $\varphi_{a b}=0$ for all $(a, b) \in A_{i, p}$.

(iii) $\mathfrak{m} H_{a}(b)=0$ for all $(a, b) \in A_{i, p}$.

Proof. By induction on $p$ and using equations (1) and (2) one proves that

$$
h_{i}(p)=\sum_{j=1}^{p-i+1}\left(\begin{array}{c}
p-j \\
i-1
\end{array}\right) \alpha_{j}-\sum_{(a, b) \in A_{i, p}}\left(\begin{array}{c}
p-b \\
i-a
\end{array}\right) \operatorname{dim}_{K} \operatorname{Im} \varphi_{a, b} .
$$


Then (a) and the equivalence of (i) and (ii) in (b) follow immediately. For the equivalence of (ii) and (iii) we notice that a generic linear form annihilates $H_{a}(b)$ if and only if $\mathfrak{m} H_{a}(b)=0$.

By taking $p=n$ we obtain:

Corollary 1.2. (a) $\beta_{i} \leq \sum_{j=1}^{n-i+1}\left(\begin{array}{c}n-j \\ i-1\end{array}\right) \alpha_{j}$ for all $i \geq 1$.

(b) For a given $i$ the following conditions are equivalent:

(i) $\beta_{i}=\sum_{j=1}^{n-i+1}\left(\begin{array}{c}n-j \\ i-1\end{array}\right) \alpha_{j}$,

(ii) $\mathfrak{m} H_{a}(b)=0$ for all $(a, b) \in A_{i, n}$.

(c) The following conditions are equivalent:

(i) $\beta_{i}=\sum_{j=1}^{n-i+1}\left(\begin{array}{c}n-j \\ i-1\end{array}\right) \alpha_{j}$ for all $i \geq 1$,

(ii) $\mathfrak{m} H_{a}(b)=0$ for all $b$ and for all $a \geq 1$.

We now want to discuss when condition (c)(ii) is satisfied. We first note that it implies that $y_{1}, \ldots, y_{n}$ is a proper sequence in the sense of [13].

Definition 1.3. Let $R$ be an arbitrary commutative ring, and $M$ and $R$-module. A sequence $y_{1}, \ldots, y_{r}$ of elements of $R$ is called a proper $M$-sequence, if $y_{p+1} H_{i}(p ; M)=0$ for all $i \geq 1$ and $p=0, \ldots, r-1$.

In [16] Kühl proved the following remarkable fact: The sequence $y_{1}, \ldots, y_{r}$ is a proper $R$-sequence if and only if

$$
y_{p+1} H_{1}(p, R)=0 \quad \text { for } \quad p=0, \ldots, r-1 .
$$

Let $I$ be a graded ideal of $S$, then we write $I_{\langle j\rangle}$ for the ideal generated by all homogeneous polynomials of degree $j$ belonging to $I$.

A homogeneous ideal $I \subset S$ is called componentwise linear [14] if $I_{\langle j\rangle}$ has a linear resolution for all $j$.

For a monomial $u \in S$ we set

$$
m(u)=\max \left\{i: x_{i} \mid u\right\} .
$$

Recall that a monomial ideal $I \subset S$ is strongly stable if, for all monomials $u$ belonging to $I$ and all for all variables $x_{j}$ which divide $u$, one has $x_{i}\left(u / x_{j}\right) \in I$ for all $i<j$. Moreover $I$ is called stable if $x_{i}\left(u / x_{m(u)}\right) \in I$ for all monomials $u \in I$ and all $i<m(u)$. The minimal free resolution of a stable ideal has been described by Eliahou and Kervaire [12]. If a monomial ideal $I \subset S$ is stable, then $I_{\langle j\rangle}$ is stable for all $j$. It follows then from the result in [12] that $I_{\langle j\rangle}$ has a linear resolution (independent of the characteristic of $K$ ). Hence a stable ideal is componentwise linear.

Let $\operatorname{Gin}(I)$ denote the generic initial ideal of $I$ with respect to the reverse lexicographical order induced by $x_{1}>x_{2}>\ldots>x_{n}$. In general $\operatorname{Gin}(I)$ is Borel-fixed, 
i.e. it is invariant under the action of the upper triangular invertible matrices, see [10]. Any strongly stable ideal is Borel-fixed and the converse is true in characteristic 0. In prime characteristic the combinatorial description of the Borel-fixed ideals is more complicated, nevertheless one has:

Lemma 1.4. In arbitrary characteristic, if I is componentwise linear, then $\operatorname{Gin}(I)$ is stable.

Proof. Since $I_{\langle j\rangle}$ has a linear resolution, it follows that $\operatorname{reg} I_{\langle j\rangle}=j$. Here $\operatorname{reg} M$ denotes the regularity of a graded $S$-module $M$. By the Bayer-Stillman theorem, cf. [5] or [10] we have reg $\operatorname{Gin}\left(I_{\langle j\rangle}\right)=j$, too. Now we apply a result of Eisenbud, Reeves and Totaro [11, Proposition 10]. It says that reg $\operatorname{Gin}(I)$ is the largest integer $j$ such that $\beta_{0 j}(\operatorname{Gin}(I)) \neq 0$ and $\operatorname{Gin}(I)_{j}$ generates a stable ideal. Hence we conclude that $\operatorname{Gin}\left(I_{\langle j\rangle}\right)_{j}$ generates a stable ideal. Thus, since $\operatorname{Gin}(I)_{j}=\operatorname{Gin}\left(I_{\langle j\rangle}\right)_{j}$, the assertion follows.

To state the next theorem we need one more definition: Let $M$ be a graded $S$-module and $G$ the minimal graded free $S$-resolution of $M$. We set $\mathcal{F}_{j}\left(G_{i}\right)=$ $\mathfrak{m}^{j-i} G_{i}$ for all $i$ and $j$. Then $(G, \mathcal{F})$ is a filtered complex whose associated graded complex we denote by $\operatorname{gr}_{\mathfrak{m}}(G)$. Note that $\operatorname{gr}_{\mathfrak{m}}(G)$ can be be identified with the complex of free modules which is obtained from $G$ by replacing in the matrices representing the differentials of $G$ all entries of degree $>1$ by 0 . One calls $\operatorname{gr}_{\mathfrak{m}}(G)$ the linear part of $G$. The largest integer $i$ for which $H_{i}\left(\operatorname{gr}_{\mathfrak{m}}(G)\right) \neq 0$ is said to be the number where the linear part of $G$ predominates. We denote it by $\operatorname{lpd}(M)$. Note that $\operatorname{lpd}(M)=0$ is equivalent to the fact that $\operatorname{gr}_{\mathfrak{m}}(G)$ is an acyclic free complex.

Theorem 1.5. Assume that $\operatorname{char}(K)=0$, and let $I \subset S$ be a graded ideal. Set $R=S / I$, and let $y=y_{1}, \ldots, y_{n}$ be a sequence of generic linear forms. The following conditions are equivalent:

(a) $R$ has maximal Betti numbers, i.e.

$$
\beta_{i}(R)=\sum_{j=1}^{n-i+1}\left(\begin{array}{c}
n-j \\
i-1
\end{array}\right) \alpha_{j}(R) \quad \text { for all } i \geq 1
$$

(b) y is a proper $R$-sequence;

(c) I is componentwise linear;

(d) I and $\operatorname{Gin}(I)$ have the same Betti numbers;

(e) $\operatorname{lpd}(I)=0$.

Proof. Let $z$ be a generic linear form. Then $z H_{i}(p)=0$ if and only if $\mathfrak{m} H_{i}(p)=0$. Thus the equivalence of (a) and (b) follows from 1.2 (c). The equivalence of (b) and (c) can be found in [8, Theorem 4.4]. The equivalence of (c) and (d) is the content of [2, Theorem 1.1], while the equivalence of (d) and (e) has been shown 
by Römer in his dissertation [17, Theorem 3.2.8].

Notice that Theorem 1.5 applies in particular to the case when $I$ is a stable ideal. Here the generic annihilators $\alpha_{i}(R)$ of $R=S / I$ have an explicit interpretation. Given a monomial ideal $I$ of $S$ we write $G(I)$ for the unique minimal system of monomial generators of $I$. Let $m_{i}(I)$ denote the number of monomials $u \in G(I)$ with $m(u)=i$, and set $m_{\leq i}(I)=\sum_{j=1}^{i} m_{j}(I)$. If a monomial ideal $I \subset S$ is stable, then

$$
\beta_{i}(I)=\sum_{u \in G(I)}\left(\begin{array}{c}
m(u)-1 \\
i
\end{array}\right)=\sum_{j=i+1}^{n} m_{j}(I)\left(\begin{array}{c}
j-1 \\
i
\end{array}\right)
$$

for all $i$, see [12]. By arguing directly or by comparing (3) with 1.2 (a) we see that

$$
\alpha_{i}(R)=m_{n-i+1}(I) \quad \text { for } \quad i=1, \ldots, n .
$$

Remark 1.6. Let $(R, \mathfrak{m})$ be a regular local ring, and $M$ a finitely generated $R$ local. Assuming that the residue class field is infinite, regular system of parameters $y_{1}, \ldots, y_{n}$ can be chosen such that $A_{p}=\left(y_{1}, \ldots, y_{p-1}\right) M:_{M} y_{p} /\left(y_{1}, \ldots, y_{p-1}\right) M$ is of finite length. Denoting by $\alpha_{p}$ the length of $A_{p}$ it is easy to see that the conditions (a), (b) and (e) of Theorem 1.5 are equivalent in the local case, too.

\section{Rigidity of resolutions}

In this section we will show that the tail of a resolution has a rigid behavior with respect to big Betti numbers. For the proof of this result we need a lemma on the vanishing of Koszul homology.

Let $R$ be an arbitrary commutative ring and $M$ an $R$-module. For a sequence $y_{1}, \ldots, y_{r} \in R$ and a subset $A \subseteq\{1, \ldots, r\}$, we set $y_{A}=\left\{y_{j}: j \in A\right\}$, and for any $j \in A$ we set $A_{j}=A \backslash\{j\}$.

For all $i$ with $1 \leq i \leq r$ and $j \in A$ there is a canonical map

$$
\partial_{j}: H_{i+1}\left(y_{A} ; M\right) \longrightarrow H_{i}\left(y_{A_{j}} ; M\right)
$$

defined as follows: let $[z] \in H_{i+1}\left(y_{A} ; M\right)$ be the homology class of a cycle $z \in$ $Z_{i+1}\left(y_{A} ; M\right)$. The cycle $z$ can uniquely be written as $z=z_{0}+z_{1} \wedge e_{j}$, where $z_{0} \in K_{i+1}\left(y_{A_{j}} ; M\right)$ and $z_{1} \in Z_{i}\left(y_{A_{j}} ; M\right)$. We set $\partial_{j}([z])=\left[z_{1}\right]$. Note that $\partial_{j}$ appears in the long exact sequence

$$
\cdots \longrightarrow H_{i+1}\left(y_{A_{j}} ; M\right) \longrightarrow H_{i+1}\left(y_{A} ; M\right) \stackrel{\partial_{j}}{\longrightarrow} H_{i}\left(y_{A_{j}} ; M\right) \stackrel{y_{j}}{\longrightarrow} \cdots
$$

Finally we let

$$
\partial: H_{i+1}\left(y_{A} ; M\right) \longrightarrow \bigoplus_{j \in A} H_{i}\left(y_{A_{j}} ; M\right)
$$

be the canonical map with $\partial([z])=\left(\partial_{j}([z])\right)_{j \in A}$. 
Lemma 2.1. Let $i>0$ be an integer. Assume that for all $A \subseteq\{1, \ldots, r\}$ and all $s>\max A$ with $s \leq r$ one has $y_{s} H_{i}\left(y_{A} ; M\right)=0$. Then for all $A \subseteq\{1, \ldots, r\}$ the canonical map

$$
\partial: H_{i+1}\left(y_{A} ; M\right) \longrightarrow \bigoplus_{j \in A} H_{i}\left(y_{A_{j}} ; M\right)
$$

is injective.

Proof. We proceed by induction on $|A|$. Let $k=\max A$ and $B=A \backslash\{k\}$. We then obtain a commutative diagram

$$
\begin{aligned}
& H_{i+1}\left(y_{B} ; M\right) \stackrel{\partial}{\longrightarrow} \bigoplus_{j \in A, j \neq k} H_{i}\left(y_{B_{j}} ; M\right) \\
& \quad \downarrow \\
& H_{i+1}\left(y_{A} ; M\right) \stackrel{\partial}{\longrightarrow} \bigoplus_{j \in A, j \neq k} H_{i}\left(y_{A_{j}} ; M\right) \oplus H_{i}\left(y_{A_{k}} ; M\right) .
\end{aligned}
$$

Here the vertical maps are the natural ones.

Let $v \in H_{i+1}\left(y_{A} ; M\right)$ and suppose that $\partial(v)=0$. Then in particular $\partial_{k}(v)=0$, and hence there exists $w \in H_{i+1}\left(y_{B} ; M\right)$ such that $g(w)=v$. Since the diagram is commutative we get $f(\partial(w))=0$.

By the induction hypothesis $\partial: H_{i+1}\left(y_{B} ; M\right) \rightarrow \bigoplus_{j \in A, j \neq k} H_{i}\left(y_{B_{j}} ; M\right)$ is injective, and our assumption implies that $y_{k} H_{i}\left(y_{B_{j}} ; M\right)=0$ for all $j \in A$ with $j \neq k$, so that the map $f$ is injective, too. It follows that $w=0$, and hence $v=g(w)=0$.

Corollary 2.2. Let $I \supseteq\left(y_{1}, \ldots, y_{r}\right)$ and assume that $I H_{i}\left(y_{A} ; M\right)=0$ for all $A \subseteq\{1, \ldots, r\}$. Then $I H_{i+1}\left(y_{A} ; M\right)=0$ for all $A \subseteq\{1, \ldots, r\}$.

We remark that a related result can be deduced from the theorem of Kühl quoted in Section 1: Set $J=\left(y_{1}, \ldots, y_{r}\right)$ and assume that for a given $i$ one has $J H_{i}(p ; M)=0$ for $p=1, \ldots, r-1$, then $J H_{i+1}(p ; M)=0$ for $=1, \ldots, r-1$.

Theorem 2.3. Let $M$ be a graded $S$-module. Suppose $\beta_{i}(M)=\sum_{j=1}^{n-i+1}\left(\begin{array}{c}n-j \\ i-1\end{array}\right) \alpha_{j}(M)$ for some $i$. Then

$$
\beta_{k}(M)=\sum_{j=1}^{n-k+1}\left(\begin{array}{l}
n-j \\
k-1
\end{array}\right) \alpha_{j}(M) \quad \text { for all } \quad k \geq i .
$$

Proof. Clearly it is enough to prove the statement for $k=i+1$. Let $y=y_{1}, \ldots, y_{n}$ be a sequence of generic linear forms and denote by $H_{a}(b)$ the associated Koszul homology $H_{a}(b ; M)$. By Proposition 1.1(b) we have to show that $\mathfrak{m} H_{a}(b)=0$ for all $(a, b) \in A_{i, n}$ implies that $\mathfrak{m} H_{a}(b)=0$ for all $(a, b) \in A_{i+1, n}$. But

$$
A_{i+1, n} \backslash A_{i, n}=\{(i+1, b): b \leq n-1\} .
$$


Since $(i, b) \in A_{i, n}$ for all $b$ and since any permutation of $y$ is a again a generic sequence, it follows that $\mathfrak{m} H_{i}\left(y_{A} ; M\right)=0$ for any subset $A \subseteq\{1, \ldots, n\}$. Hence by Corollary 2.2 we conclude that $\mathfrak{m} H_{i+1}\left(y_{A} ; M\right)=0$ for all $A$ and in particular $\mathfrak{m} H_{i+1}(b)=0$, as desired.

The following corollary generalizes a result of Aramova, Herzog and Hibi [2], explicitly stated as Theorem 1.2 in [8].

Corollary 2.4. Assume $\operatorname{char}(K)=0$, and let $I \subset S$ be a graded ideal. Suppose that $\beta_{i}(I)=\beta_{i}(\operatorname{Gin}(I))$ for some $i$. Then

$$
\beta_{k}(I)=\beta_{k}(\operatorname{Gin}(I)) \quad \text { for all } \quad k \geq i .
$$

For the proof of this corollary we need

Lemma 2.5. Let $I \subset S$ be graded ideal. Then $\alpha_{j}(S / I)=\alpha_{j}(S / \operatorname{Gin}(I))$ for all $j$.

Proof. After a generic change of coordinates we may assume that $\operatorname{Gin}(I)=\operatorname{in}(I)$, and that $x_{n}, x_{n-1}, \ldots, x_{1}$ is a generic sequence. For the reverse lexicographical order induced by $x_{1}>x_{2}>\ldots>x_{n}$ one has

$$
\operatorname{in}\left(\left(x_{i}, \ldots, x_{n}\right)+I\right)=\left(x_{i}, \ldots, x_{n}\right)+\operatorname{in}(I)
$$

and

$$
\left.\operatorname{in}\left(\left(x_{i}, \ldots, x_{n}\right)+I\right): x_{i-1}\right)=\left(\left(x_{i}, \ldots, x_{n}\right)+\operatorname{in}(I)\right): x_{i-1} .
$$

It follows that

$$
\left(\left(x_{i}, \ldots, x_{n}\right)+I\right): x_{i-1} /\left(x_{i}, \ldots, x_{n}\right)+I
$$

and

$$
\left(\left(x_{i}, \ldots, x_{n}\right)+\operatorname{in}(I)\right): x_{i-1} /\left(x_{i}, \ldots, x_{n}\right)+\operatorname{in}(I)
$$

have the same Hilbert function. This yields the desired conclusion.

Proof of Corollary 2.4. Since we assume char $(K)=0$ the ideal Gin $(I)$ is strongly stable and hence componentwise linear. It follows from 1.5 that

$$
\beta_{i+1}(S / \operatorname{Gin}(I))=\sum_{j=1}^{n-i+2}\left(\begin{array}{c}
n-j \\
i
\end{array}\right) \alpha_{j}(S / \operatorname{Gin}(I)) .
$$

By Lemma 2.5 and our assumption this implies that

$$
\beta_{i+1}(S / I)=\sum_{j=1}^{n-i+2}\left(\begin{array}{c}
n-j \\
i
\end{array}\right) \alpha_{j}(S / I)
$$

Now we apply Theorem 2.3 and again Lemma 2.5 to conclude that

$$
\beta_{k}(I)=\beta_{k+1}(S / I)=\sum_{j=1}^{n-k+2}\left(\begin{array}{c}
n-j \\
k
\end{array}\right) \alpha_{j}(S / I)
$$




$$
\begin{aligned}
& =\sum_{j=1}^{n-k+2}\left(\begin{array}{c}
n-j \\
k
\end{array}\right) \alpha_{j}(S / \operatorname{Gin}(I)) \\
& =\beta_{k+1}(S / \operatorname{Gin}(I))=\beta_{k}(\operatorname{Gin}(I))
\end{aligned}
$$

for $k=i, \ldots, n-1$.

We give an example of an ideal $I$ (many other such examples may be constructed) for which $I$ and $\operatorname{Gin}(I)$ have different resolutions, but the tail of their resolutions are the same.

Example 2.6. Let

$$
I=\left(x_{1}^{2}, x_{2}^{2}, x_{3}^{2}\right)+\left(x_{1}, x_{2}, \ldots, x_{5}\right)^{3},
$$

then

$$
\operatorname{Gin}(I)=\left(x_{1}^{2}, x_{1} x_{2}, x_{2}^{2}\right)+\left(x_{1}, x_{2}, x_{3}\right)^{3} .
$$

The minimal free resolution of $I$ and $\operatorname{Gin}(I)$ are, respectively,

$$
\begin{aligned}
0 \rightarrow R^{12}(-7) \rightarrow R^{55}(-6) \rightarrow & R^{96}(-5) \rightarrow \\
& R^{2}(-3) \oplus R^{75}(-4) \rightarrow R^{3}(-2) \oplus R^{22}(-3) \rightarrow 0
\end{aligned}
$$

We have also:

Corollary 2.7. Assume $\operatorname{char}(K)=0$, and let $I \subset S$ be a graded ideal. Let $J$ be either the (unique) lex-segment ideal with the same Hilbert function as $I$ or the generic initial ideal of $I$ with respect to a term order $\tau$. Suppose that $\beta_{i}(I)=\beta_{i}(J)$ for some $i$. Then

$$
\beta_{k}(I)=\beta_{k}(J) \quad \text { for all } \quad k \geq i
$$

Proof. Set $G=\operatorname{Gin}(I)$. One has $\beta_{j}(I) \leq \beta_{j}(G) \leq \beta_{j}(J)$ for all $j$. This is due to Bigatti [4] and Hullett [15] when $J$ is the lex-segment ideal and to Conca [8, Theorem 5.1.] when $J$ is a gin of $I$. Hence by Corollary 2.4 we have that $\beta_{k}(I)=\beta_{k}(G)$ for all $k \geq i$. Therefore it suffices to show that $\beta_{k}(G)=\beta_{k}(J)$ for all $k \geq i$. We have $m_{\leq i}\left(J_{\langle j\rangle}\right) \leq m_{\leq i}\left(G_{\langle j\rangle}\right)$ for all $i$ and $j$ : this is a result of Bayer [3] and Bigatti [4] when $J$ is the lex-segment ideal and a result of Conca when $J$ is a gin of $I$ (see the proof of [8, Theorem 5.1.]). This however implies $m_{i}(J) \geq m_{i}(G)$, see [8, Proposition 3.3]. Taking into account the Eliahou-Kervaire formula (3) for the Betti numbers of a stable ideals, our assumption and the inequalities $m_{j}(J) \geq m_{j}(G)$ imply $m_{j}(J)=m_{j}(G)$ for all $j>i$. Applying again the Eliahou-Kervaire formula (3) we see that $\beta_{k}(G)=\beta_{k}(J)$ for all $k \geq i$.

We conclude this section with an example of a strongly stable ideal $I$ whose 
corresponding lex-segment ideal Lex $(I)$ has a free resolution which is different from that of $I$, but has the same tail.

Example 2.8. Let

$$
I=\left(x_{1}, \ldots, x_{2}\right)^{2}+\left(x_{1} x_{2}^{2}, x_{1} x_{3} x_{4}\right) \text { in } S=K\left[x_{1}, \ldots, x_{4}\right] .
$$

The ideal $I$ is strongly stable and its Lex-segmente ideal is

$$
\operatorname{Lex}(I)=\left(x_{1}^{2}, x_{1} x_{2}, x_{1} x_{3}, x_{1} x_{4}^{2}, x_{2}^{3}, x_{2} x_{3}\right) .
$$

The minimimal free resolution of $I$ and $\operatorname{Lex}(I)$ are, respectively,

$$
\begin{aligned}
& 0 \rightarrow S(-6) \rightarrow \quad S^{4}(-5) \rightarrow S^{2}(-3) \oplus S^{5}(-4) \rightarrow S^{3}(-2) \oplus S^{2}(-3) \rightarrow 0 \\
& 0 \rightarrow S(-6) \rightarrow S(-4) \oplus S^{4}(-5) \rightarrow S^{3}(-3) \oplus S^{6}(-4) \rightarrow S^{3}(-2) \oplus S^{3}(-3) \rightarrow 0
\end{aligned}
$$

\section{Betti numbers and Hilbert polynomials}

In this section we compare the Betti numbers of two componentwise linear ideals $I \subset J$ which have the same Hilbert polynomial.

If a graded ideal $I \subset S$ is componentwise linear, then

$$
\beta_{i, i+j}(I)=\beta_{i}\left(I_{\langle j\rangle}\right)-\beta_{i}\left(\mathfrak{m} I_{\langle j-1\rangle}\right)
$$

for all $i$ and $j$, see [14, Proposition 1.3].

Let $I$ be a strongly stable ideal generated by monomials of the same degree. Then

$$
m_{i}(\mathfrak{m} I)=m_{\leq i}(I)
$$

for all $i$, see [4, Proposition 1.3].

Lemma 3.1. Let $I \subset S$ be a strongly stable ideal and fix $1 \leq d \leq N$ such that $d \leq \operatorname{deg}(u) \leq N$ for all $u \in G(I)$. Then, for all $i$, one has

$$
\beta_{i}\left(I_{\langle N+1\rangle}\right)-\beta_{i}(I)=\sum_{j=d}^{N} \sum_{k=i+1}^{n} m_{\leq k-1}\left(I_{\langle j\rangle}\right)\left(\begin{array}{c}
k-1 \\
i
\end{array}\right) .
$$

Proof. Since $I_{\langle j\rangle}$ is strongly stable, it follows from the formulae (4) and (5) that

$$
\begin{aligned}
\beta_{i}\left(I_{\langle j\rangle}\right)-\beta_{i}\left(\mathfrak{m} I_{\langle j\rangle}\right) & =\sum_{k=i+1}^{n} m_{k}\left(I_{\langle j\rangle}\right)\left(\begin{array}{c}
k-1 \\
i
\end{array}\right)-\sum_{k=i+1}^{n} m_{k}\left(\mathfrak{m} I_{\langle j\rangle}\right)\left(\begin{array}{c}
k-1 \\
i
\end{array}\right) \\
& =\sum_{k=i+1}^{n} m_{k}\left(I_{\langle j\rangle}\right)\left(\begin{array}{c}
k-1 \\
i
\end{array}\right)-\sum_{k=i+1}^{n} m_{\leq k}\left(I_{\langle j\rangle}\right)\left(\begin{array}{c}
k-1 \\
i
\end{array}\right) \\
& =-\left(\sum_{k=i+1}^{n} m_{\leq k-1}\left(I_{\langle j\rangle}\right)\left(\begin{array}{c}
k-1 \\
i
\end{array}\right)\right) .
\end{aligned}
$$


Since $I_{\langle d-1\rangle}=0$ and $\mathfrak{m} I_{\left\langle N^{\prime}\right\rangle}=I_{\left\langle N^{\prime}+1\right\rangle}$ for all $N^{\prime} \geq N$, by using the formula (4), it follows that

$$
\begin{aligned}
\beta_{i}(I) & =\sum_{j=0}^{\infty} \beta_{i, i+j}(I)=\sum_{j=d}^{N}\left(\beta_{i}\left(I_{\langle j\rangle}\right)-\beta_{i}\left(\mathfrak{m} I_{\langle j-1\rangle}\right)\right) \\
& =\sum_{j=d}^{N}\left(\beta_{i}\left(I_{\langle j\rangle}\right)-\beta_{i}\left(\mathfrak{m} I_{\langle j\rangle}\right)\right)+\beta_{i}\left(\mathfrak{m} I_{\langle N\rangle}\right) \\
& =\sum_{j=d}^{N}\left(\beta_{i}\left(I_{\langle j\rangle}\right)-\beta_{i}\left(\mathfrak{m} I_{\langle j\rangle}\right)\right)+\beta_{i}\left(I_{\langle N+1\rangle}\right) \\
& =-\sum_{j=d}^{N}\left(\sum_{k=i+1}^{n} m_{\leq k-1}\left(I_{\langle j\rangle}\right)\left(\begin{array}{c}
k-1 \\
i
\end{array}\right)\right)+\beta_{i}\left(I_{\langle N+1\rangle}\right),
\end{aligned}
$$

as desired.

We are now in the position to state the main result of the present section.

Theorem 3.2. Let $I$ and $J$ be componentwise linear ideals of $S$ with $I \subset J$, and suppose that $I$ and $J$ have the same Hilbert polynomial. Then we have:

(a) $\beta_{i}(J) \leq \beta_{i}(I)$ for all $i$.

(b) if $\beta_{i}(J)=\beta_{i}(I)$ for some $0 \leq i<n$, then $\beta_{k}(J)=\beta_{k}(I)$ for all $k$.

(c) Let $y$ be a generic linear form. Then the following conditions are equivalent:

(i) $\beta_{i}(I)=\beta_{i}(J)$ for some $0 \leq i<n$;

(ii) $I+(y)=J+(y)$.

Proof. By Lemma 1.4 the generic initial ideals $\operatorname{Gin}(I)$ and $\operatorname{Gin}(J)$ of $I$ and $J$ are stable. Since $I$ and $J$ are componentwise linear, [2, Theorem 1.1] guarantees that $\beta_{i}(I)=\beta_{i}(\operatorname{Gin}(I))$ and $\beta_{i}(J)=\beta_{i}(\operatorname{Gin}(J))$ for all $i$. In [2] it is assumed that the base field is of characteristic 0 . However for this direction one does not need this hypothesis. In fact, since $\operatorname{Gin}(I)$ is stable by Lemma 1.4 the argument in the proof of $[2$, Theorem 1.1] is valid. Since $I \subset J$, one has $\operatorname{Gin}(I) \subset \operatorname{Gin}(J)$. Therefore, in proving (a), (b) we may replace $I, J$ with their gin and assume that both $I$ and $J$ are stable. Since the resolution of a stable ideal is independent of the characteristic we may assume that the characteristic in 0 and thus taking again generic initial ideals may assume that $I$ and $J$ are even strongly stable, at least when dealing with the statements (a) and (b). When dealing with (c) we may also replace $I$ and $J$ with their gins and $y$ with $x_{n}$. This is because, $I+(y)=J+(y)$ holds if and only if the two ideals have the same Hilbert function and the Hilbert function of $I+(y)$ does not change by replacing $I$ with $\operatorname{Gin}(I)$ and $y$ with $x_{n}$. Note that a stable ideal is invariant under any linear transformation $h$ with $h\left(x_{i}\right)=x_{i}$ for all $i=1, \ldots, n-1$. It follows that $x_{n}$ is a generic linear form with respect to a stable 
ideal. So the equality $I+\left(x_{n}\right)=J+\left(x_{n}\right)$ can be checked in any characteristic and we may assume that the characteristic is 0 and take the gin again. Summing up, for all the three statements, we may assume that $I$ and $J$ are strongly stable monomial ideals and that $y$ is $x_{n}$.

(a) Since $I \subset J$, and since $I$ and $J$ have the same Hilbert polynomial, there is $N>0$ with $\mathfrak{m} I_{\langle N\rangle}=I_{\langle N+1\rangle}=J_{\langle N+1\rangle}=\mathfrak{m} J_{\langle N\rangle}$ Let $d=\min \left\{d_{I}, d_{J}\right\}$, where $d_{I}=$ $\min \{\operatorname{deg}(u): u \in G(I)\}$ and $d_{J}=\min \{\operatorname{deg}(v): v \in G(J)\}$. Then $d \leq \operatorname{deg}(w) \leq N$ for all $w \in G(I) \cup G(J)$. Now, Lemma 3.1 guarantees that

$$
\beta_{i}(I)-\beta_{i}(J)=\sum_{j=d}^{N} \sum_{k=i+1}^{n}\left(m_{\leq k-1}\left(J_{\langle j\rangle}\right)-m_{\leq k-1}\left(I_{\langle j\rangle}\right)\right)\left(\begin{array}{c}
k-1 \\
i
\end{array}\right) .
$$

Since $I \subset J$, one has $G\left(I_{\langle j\rangle}\right) \subset G\left(J_{\langle j\rangle}\right)$ for all $j$. It then follows that

$$
m_{i}\left(I_{\langle j\rangle}\right) \leq m_{i}\left(J_{\langle j\rangle}\right), \quad m_{\leq i}\left(I_{\langle j\rangle}\right) \leq m_{\leq i}\left(J_{\langle j\rangle}\right)
$$

for all $j$ and for all $i$. Thus $\beta_{i}(J) \leq \beta_{i}(I)$ for all $i$.

(b) If $\beta_{i}(J)=\beta_{i}(I)$ for some $0 \leq i<n$, then $m_{\leq k-1}\left(I_{\langle j\rangle}\right)=m_{\leq k-1}\left(J_{\langle j\rangle}\right)$ for all $j$ and for all $i<k \leq n$. Thus in particular $m_{\leq n-1}\left(I_{\langle j\rangle}\right)=m_{\leq n-1}\left(J_{\langle j\rangle}\right)$ for all $j$.

Since $m_{\leq n-1}\left(I_{\langle j\rangle}\right)=\sum_{k=1}^{n-1} m_{k}\left(I_{\langle j\rangle}\right)$ and $m_{\leq n-1}\left(J_{\langle j\rangle}\right)=\sum_{k=1}^{n-1} m_{k}\left(J_{\langle j\rangle}\right)$, and since $m_{k}\left(I_{\langle j\rangle}\right) \leq m_{k}\left(J_{\langle j\rangle}\right)$, one has $m_{k}\left(I_{\langle j\rangle}\right) \stackrel{=}{=} m_{k}\left(J_{\langle j\rangle}\right)$ for all $j$ and for all $k \leq n-1$. Hence $\beta_{k}(J)=\beta_{k}(I)$ for all $k$.

(c) Let $\bar{I}=I+\left(x_{n}\right) /\left(x_{n}\right)$ and $\bar{J}=J+\left(x_{n}\right) /\left(x_{n}\right)$. Then $\bar{I}$ and $\bar{J}$ are strongly stable ideals in $K\left[x_{1}, \ldots, x_{n-1}\right]$ with $m_{k}\left(I_{\langle j\rangle}\right)=m_{k}\left(\bar{I}_{\langle j\rangle}\right)$ and $m_{k}\left(J_{\langle j\rangle}\right)=m_{k}\left(\bar{J}_{\langle j\rangle}\right)$ for all $j$ and all $k \leq n-1$. In the proof of (b) we have seen that $\beta_{i}(I)=\beta_{i}(J)$ for some $i$, if and only if $m_{\leq n-1}\left(I_{\langle j\rangle}\right)=m_{\leq n-1}\left(J_{\langle j\rangle}\right)$ for all $j$. But this is the case if and only if $\bar{I}$ and $\bar{J}$ have the same Hilbert function. This in turn is equivalent to saying that $\bar{I}=\bar{J}$.

Remark 3.3. If in Theorem 3.2 we assume that $I$ and $J$ are strongly stable with the same Hilbert polynomial, then the assumption $I \subset J$ may be replaced by the weaker assumption $m_{\leq i}\left(I_{j}\right) \leq m_{\leq i}\left(J_{j}\right)$ for all $i$ and $j$ in order to conclude 3.2.(a), and by $m_{i}\left(I_{j}\right) \leq m_{i}\left(J_{j}\right)$ for all $i$ and $j$ in order to conclude 3.2.(b).

Let $y \in S$ be a generic linear form. For any ideal $J \subset S$ we denote by $\bar{J}$ the image of $J$ in $\bar{S}=S / y S$.

Corollary 3.4. Assume char $(K)=0$, and let $I \subset S$ be an $\mathfrak{m}$-primary graded ideal. Suppose that $I \subset \mathfrak{m}^{d}$. Then

(a) $\beta_{0}(\operatorname{Gin}(I)) \geq\left(\begin{array}{c}n+d-1 \\ d\end{array}\right)$.

(b) $\beta_{0}(\operatorname{Gin}(I))=\left(\begin{array}{c}n+d-1 \\ d\end{array}\right)$ if and only if $\bar{I}=\overline{\mathfrak{m}}^{d}$.

Similarly we obtain also an upper bound for the number of generators of $\operatorname{Gin}(I)$ : 
Corollary 3.5. Assume char $(K)=0$, and let $I \subset S$ be an $\mathfrak{m}$-primary graded ideal generated in degree $d$. Let $C$ be the ideal generated by a regular sequence of $n$ elements of degree $d$ in $I$. Then $\beta_{0}(\operatorname{Gin}(I)) \leq \beta_{0}(\operatorname{Gin}(C))$.

Proof. The ideals $\operatorname{Gin}(C)$ and $\operatorname{Gin}(I)$ are strongly stable and hence componentwise linear. Furthermore, they have the same Hilbert polynomial (since they are both Artinian) and $\operatorname{Gin}(C) \subseteq \operatorname{Gin}(I)$. The conclusion then follows from 3.2.

In view of this result one might ask whether the gin of a complete intersection does depend on the specific complete intersection. Not surprisingly, it does. For instance in the case $d=3$ and $n=4$ the monomial and the generic complete intersection have distinct gins but the two ideals have the same Betti numbers. For $d=3$ and $n=5$ the monomial and the generic complete intersection have distinct gins and the gin of the monomial c.i. has 77 generators while that of the generic c.i. has "only" 76 generators. It would be nevertheless interesting to have an upper bound for the number of generators of $\operatorname{Gin}(I)$ which just depend on the $n$ and $d$. To this end, the following question is of interest: Let $f_{1}, \ldots, f_{n}$ be a regular sequence of forms of degree $d$ in $n$ variables. Is it true that $\beta_{0}\left(\operatorname{Gin}\left(f_{1}, \ldots, f_{n}\right)\right) \leq$ $\beta_{0}\left(\operatorname{Gin}\left(x_{1}^{d}, \ldots, x_{n}^{d}\right)\right)$ ? What we can prove is the following:

Lemma 3.6. Assume $\operatorname{char}(K)=0$. Let $I$ be a generic complete intersection of $n$ forms of degree $d$ in $K\left[x_{1}, \ldots, x_{n}\right]$ and let $J=\left(x_{1}^{d}, \ldots, x_{n}^{d}\right)$. Then $\beta_{i j}(\operatorname{Gin}(I)) \leq$ $\beta_{i j}(\operatorname{Gin}(J))$.

Proof. Let $I=\left(f_{1}, \ldots, f_{n}\right)$ where $f_{1}, \ldots, f_{n}$ is a regular sequence of forms of degree $d$. Consider the ideal $H$ of $K\left[x_{1}, \ldots, x_{n}, y_{1}, \ldots, y_{n}\right]$ generated by $g_{i}=f_{i}+y_{i}^{d}$, and let $H^{\prime}$ be the ideal generated by $f_{i}+L_{i}^{d}$ where $L_{i}$ are generic linear forms in the $x_{i}$ 's. Consider the revlex order with respect to $x_{1}>\cdots>x_{n}>y_{1}>\cdots>y_{n}$ and let $h$ be the linear map, an involution, sending $x_{i}$ to $y_{i}$ and vice versa. Then $J$ is the initial ideal of $h(H)$. Since $\operatorname{Gin}(H)=\operatorname{Gin}(h(H))$ it follows from [8, Corollary 1.6] that $m_{\leq i}\left(\operatorname{Gin}(H)_{j}\right) \geq m_{\leq i}\left(\operatorname{Gin}(J)_{j}\right)$ and hence $\beta_{i j}(\operatorname{Gin}(H)) \geq \beta_{i j}(\operatorname{Gin}(J))$, [8, Proposition 3.6]. But if $U$ is an ideal with $\operatorname{depth} S / U \geq k$, then $\operatorname{Gin}(U)$ does not change by factoring out $k$ generic linear forms. We get that $\operatorname{Gin}(H)=\operatorname{Gin}\left(H^{\prime}\right)$. So we have shown that $\beta_{i j}\left(\operatorname{Gin}\left(H^{\prime}\right)\right) \geq \beta_{i j}(\operatorname{Gin}(J))$. But if the $f_{i}$ are generic then the $f_{i}+L_{i}^{d}$ are generic as well.

\section{References}

[1] A. Aramova and J. Herzog, Almost regular sequences and Betti numbers, Amer. J. Math. 122 (2000), 689-719.

[2] A. Aramova, J. Herzog and T. Hibi, Ideals with stable Betti numbers, Adv. Math. 152 (2000), $72-77$. 
[3] D. Bayer, The division algorithm and the Hilbert scheme, PhD Thesis, Harvard University, 1982.

[4] A. Bigatti, Upper bounds for Betti numbers of a given Hilbert function, Comm. Alg. 21 (1993), 2317-2334

[5] D. Bayer and M. Stillman, A criterion or detecting m-regularity, Invent. Math. 87 (1987), $1-11$.

[6] W. Bruns and J. Herzog, Cohen-Macaulay rings, Revised Edition, Cambridge University Press, Cambridge, 1996

[7] A. Capani, G. Niesi and L. Robbiano, CoCoA, a system for doing Computations in Commutative Algebra, Available from cocoa.dima.unige.it.

[8] A. Conca, Koszul homology and extremal properties of Gin and Lex, to appear in Trans. $A M S$.

[9] A. Conca, Reduction numbers and initial ideals, Proc. Amer. Math. Soc. 131, no. 4 (2003), 1015-1020.

[10] D. Eisenbud, Commutative Algebra with a view to Algebraic geometry, Springer-Verlag, 1995.

[11] D. Eisenbud, A. Reeves and B. Totaro, Initial ideals, Veronese subrings, and rates of algebras, Adv. Math. 109 (1994), 168-187.

[12] S. Eliahou and M. Kervaire, Minimal resolutions of some monomial ideals, J. Algebra 129, (1990), 1-25.

[13] J. Herzog, A. Simis and W. Vasconcelos, Approximation complexes of blowing-up rings II, J. Algebra 82 (1983), 53-83.

[14] J. Herzog and T. Hibi, Componentwise linear ideals, Nagoya Math. J. 153 (1999), 141-153.

[15] H. Hulett, Maximal Betti numbers of homogeneous ideals with a given Hilbert function, Comm. Alg. 21 (1993), 2335-3250.

[16] M. Kühl, On the symmetric algebra of an ideal, manuscripta math. 7 (1982), 49-60.

[17] T. Römer, PhD Thesis, Universität Essen, 2000.

[18] N. V. Trung, Reduction exponent and degree bound for the defining equations of graded rings, Proc. Amer. Math. Soc. 101 (2) (1987), 229-236.

[19] N. V. Trung, Gröbner bases, local cohomology and reduction number, Proc. Amer. Math. Soc. 129 (1) (2001), 9-18.

Aldo Conca

DIMA - Dipartimento di Matematica

Via Dodecaneso 35

16146 Genova

Italy

e-mail: conca@dima.unige.it

Takayuki Hibi

Department of Mathematics

Graduate School of Science

Osaka University

Toyonaka

Osaka 560-0043

Japan

e-mail: hibi@math.sci.osaka-u.ac.jp

\section{Jürgen Herzog}

Fachbereich Mathematik und Informatik

Universität Duisburg-Essen

Campus Essen

45117 Essen

Germany

e-mail: juergen.herzog@uni-essen.de

(Received: June 6, 2003) 